\title{
Diastasis recti abdominis - a review of treatment methods
}

\author{
Agata Michalska1, Wojciech Rokita², Daniel Wolder², Justyna Pogorzelska1, \\ Krzysztof Kaczmarczyk ${ }^{3}$ \\ ${ }^{1}$ Institute of Physiotherapy, Jan Kochanowski University in Kielce, Poland \\ ${ }^{2}$ Department of Obstetrics and Gynecology, Voivodship Hospital in Kielce, Poland \\ ${ }^{3}$ Department of General Surgery, Hospital of the Ministry of Internal Affairs and Administration in Kielce, Poland
}

\begin{abstract}
Diastasis recti abdominis is a condition in which both rectus abdominis muscles disintegrate to the sides, this being accompanied by the extension of the linea alba tissue and bulging of the abdominal wall. DRA may result in the herniation of the abdominal viscera, but it is not a hernia per se. DRA is common in the female population during pregnancy and in the postpartum period. There is a scant knowledge on the prevalence, risk factors, prevention or management of the abovementioned condition. The aim of this paper is to present the methods of DRA treatment based on the results of recent studies.
\end{abstract} Key words: diastasis recti abdominis, conservative treatment, surgical treatment, physiotherapy

Ginekologia Polska 2018; 89, 2: 97-101

\section{INTRODUCTION}

The anterior-lateral abdominal wall is formed by muscles that may be found symmetrically on either side of the linea alba. These are, situated in the anterior median line, rectus abdominis muscles with the fibres running vertically and lateral flat muscles: external oblique, internal oblique and transversus, with obliquely and perpendicularly running fibers respectively. The tonic activity of the abdominal muscles supports and protects the viscera; it is essential for maintaining proper posture, including stabilization of the pelvis and lumbar spine. Shortening of the diagonal and transverse fibres results in increased intraabdominal pressure during coughing, laughter, micturition, defecation or childbirth. Abdominal muscles are also involved in the bending motion of the trunk (all of the abovementioned ones, and the rectus abdominis muscle, in particular, during the movement with resistance), lateral flexion (ipsilateral oblique muscles with a slight participation of the ipsilateral rectus abdominis muscle), trunk rotation (the external oblique muscle and contralateral internal oblique muscle). When the aforementioned muscles shrink during exhalation, they cause the diaphragm to lift which supports the breathing process [1].
Connected aponeuroses of the muscles of the lateral abdominal wall form a sheath that encompasses the rectus abdominis muscle. The aponeuroses of opposing sides cross in the medial line, fusing the medial borders of the sheaths. In such a way they form the linea alba (LA) running from the xiphoid process of the sternum to the superior pubic ligament. The LA performs an important role in maintaining the stability of the abdominal wall from a mechanical point of view. Its tension is regulated by pyramidalis muscles anterior to the rectus abdominis above the pubic symphysis (they do not occur permanently) [2]. There are three different zones of fibre orientation in the LA. Listing from the ventral to dorsal, these are the lamina fibrae obliquae, the lamina fibrae transversae and the small lamina fibrae irregularium. The transverse fibres act as a counterpart to the intraabdominal pressure, whereas the oblique fibers are involved mainly in movements of the trunk. There exist sex-dependent differences in the fibre architecture. In females a larger number of transverse fibres relative to oblique fibres in infraumbilical regions and a smaller thickness and increased width of the infraumbilical LA may be observed. The possible morphological differences in the LA may be 
a form of adaptation to the increasing intraabdominal pressure with pregnancy $[2,3]$.

During pregnancy, the geometry of abdominal muscles changes still maintaining their function. The expanding uterus affects the shape of the abdomen and the lumbar spine position (deepening of the lumbar lordosis) which results in an increase in the distance between the attachments, i.e. the elongation of the abdominal muscles and the change in the angle of the attachment. Functionally, this is manifested by the reduction of strength, and mainly affects the rectus abdominis muscles [1]. Besides elongation of the rectus abdominis muscles, it may lead to the stretching and flaccidity of the linea alba which may result in the enlargement of the distance between medial borders of the muscles, and a subsequent loss of their straightforward course.

\section{DIASTASIS RECTI ABDOMINIS}

An impairment characterized by the separation of the two rectus abdominis muscles along the linea alba has been defined as diastasis recti abdominis (DRA). However, in literature, other terms are sometimes used, such as rectus abdominis diastasis (RAD) or divarication of rectus abdominal muscles (DRAM) $[4,5]$.

The separation of the linea alba in DRA results in the forming of a space referred to as an inter-recti distance (IRD) (Fig. 1) [5]. According to Beer et al. [6] physiological parameters of the width of the LA amount to $15 \mathrm{~mm}$ at the height of the xiphoid process, $22 \mathrm{~mm}$ at $3 \mathrm{~cm}$ above the umbilicus and $16 \mathrm{~mm}$ at $2 \mathrm{~cm}$ below the umbilicus. The width of the LA increases with age. There is no agreement as to the size of IRD considered to be pathological. The following parameters are given: $10 \mathrm{~mm}$ above the umbilicus, $27 \mathrm{~mm}$ at the umbilical ring and $9 \mathrm{~mm}$ below the umbilicus (below 45 years of age) and $15 \mathrm{~mm}, 27 \mathrm{~mm}$ and $14 \mathrm{~mm}$ respectively (above 45 years of age) [6], above $2 \mathrm{~cm}$ [7], and in some older studies a less precise parameter - above the 2 - finger width when measured in a crook lying position.

In the clinical practice, different measurement methods of IRD are applied. There are no strict recommendations as to the place of measurement (distance from the umbilicus), the body position (at rest in the supine position or during contraction in the head lift position) or the method. The following may be applied: palpation, tape measure, calipers, ultrasound, CT and MRI [8-10]. Basing on the results analysis of thirteen studies evaluating measurement properties it was suggested that the ultrasound and calipers should be adequate methods to assess DRA [10].

The following are considered to be the risk factors of DRA: pregnancy (the resulting hormonal changes, the increased size of the uterus, anterior pelvic tilt with or without lumbar hyperlordosis, the increased intraabdominal

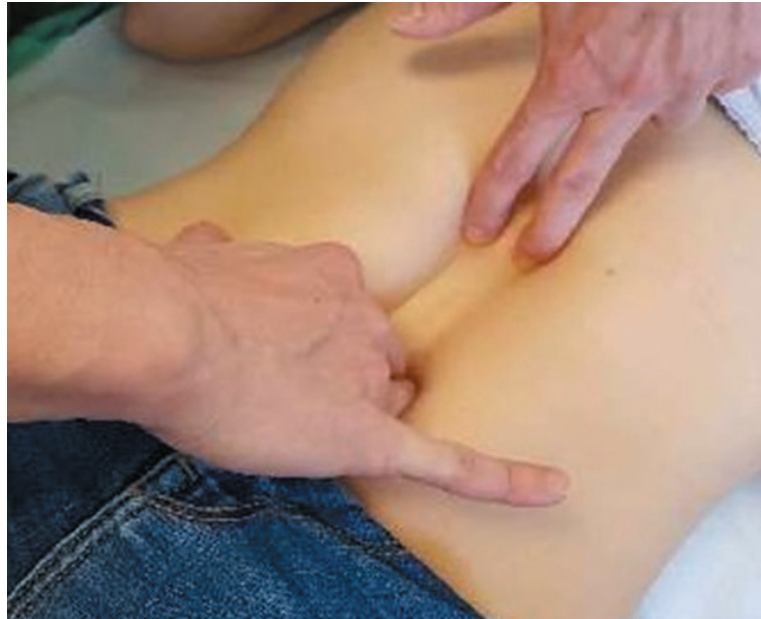

Figure 1. Clinical picture of DRA, a 30-year-old patient, 6 months postpartum (from the authors' own archives, reprinted with the patient's permission)

pressure), cesarean section, multiple pregnancies, fetal macrosomia, as well as genetically-conditioned defects in collagen structure (including congenital disproportion of the collagen III/I ratio), considerable body mass losses occurring spontaneously or after bariatric surgeries, abdominal surgical procedures [2, 3, 11, 12]. Mota et al. [13] and Sperstad et al. [14] reported no association between DRA and the pre-pregnancy body mass index, weight gain, a baby's birth weight or abdominal circumference, heavy lifting, lifting and carrying children, and regular exercise.

DRA can occur in both genders, across age groups, but it is principally seen in postpartum women. The separation between the abdominal muscles can be seen in newborns or infants as a result of the reduced abdominal muscles activity. It usually disappears spontaneously. In case of congenital, abnormal anatomy of the fibers of the LA, infants may develop a hernia, manifested by the presence of a distal, non-painful bulge located in the midline of the body between the umbilicus and the xiphoid process (more often over the umbilicus, probably due to the upward pulling of the LA by the attachments of the diaphragm) [15]. DRA may also occur in men and is thought to be associated with the increasing age, weight fluctuations, weightlifting, full sit-ups, familial weakness of abdominal muscles. The male pattern of DRA more frequently occurs primarily over the umbilicus in the fifth and sixth decades of life [16].

In the female population, DRA is common with pregnant and postpartum women. The female pattern of DRA is centered at the level of the umbilicus, but it can extend to and encompass the supraumbilical or infraumbilical region [17]. It can persist in approximately $24-70 \%$, even $100 \%$ of cases, during the postpartum period at different sites along the LA [13,17-19]. In Brazilian study the prevalence of DRA immediately after vaginal delivery was $68 \%$ above the umbilicus and 
$32 \%$ below the umbilicus. The prevalence of DRA above the umbilicus among primigravida and multiparae was identical (68\%), and the prevalence below the umbilicus was greater among multiparae (19.8\% and 29.2\%) [11].

The inter-recti distance resolves gradually with time in the postpartum period with individual variability, usually to approximately eighth week postpartum [13]. Mota et al. [13] assessed IRD and prevalence of DRA in case of 84 women in $35^{\text {th }}$ gestational week $6-8,12-14$ weeks postpartum and 6 months postpartum. The size of IRD decreased from $64.6 \mathrm{~mm}$ (SD 19.00) to $15.3 \mathrm{~mm}$ (SD 8.4), similarly the prevalence of DRA decreased from $100 \%$ to $39.3 \%$. A higher percentage of DRA occurrence at 6 months postpartum was reported by Sperstad et al. [14]. In the group of 300 women it amounted to $45.4 \%$, and decreased to $32.6 \%$ at 12 months postpartum.

An increase in the distance between the anterior borders of the rectus muscles influences the strength of the abdominal wall musculature and does not usually cause pain at rest. During physical activities, however, the characteristic bulging of the abdominal wall may appear, this being due to an increase of the intraabdominal pressure (Fig. 2). This is why DRA may be associated with epigastric and umbilical hernias [16].

Taking into account the role of abdominal muscles in maintaining posture and their engagement in various physical activities one may suspect that the presence of DRA may have an impact on the trunk and pelvic stabilization, and can lead to poor posture, limitations during physical activity, as well as lumbo-pelvic pain and hip pain [3, 20,21]. Some studies, however, deny these statements. Sperstad et al. [14] reported no difference between women with and without DRA in prevalence of low back pain, similarly as Mota et al. [13] and Parker et al. [21]. In Norwegian study, women with DRA did not have a weaker pelvic floor muscle or more pelvic floor dysfunctions than women without diastasis [22]. Other study, however, do confirm the relationship between the presence of DRA and the support-related pelvic floor dysfunction diagnoses [23].

\section{TREATMENT OF DRA}

In the majority of women DRA resolves spontaneously in the postpartum period. In case of the persistence of the inter-recti distance, the conservative treatment is applied, namely physiotherapy. It is considered to be the only treatment method that may potentially result in the decrease of IRD. Although numerous studies confirm the positive influence of exercises on reducing the inter-recti distance $[4,17,24-28]$ no generally acceptable protocol of therapeutic exercises has been formulated so far. It has not been assessed which abdominal exercises are the most effective. The most commonly applied are: an abdominal exercise

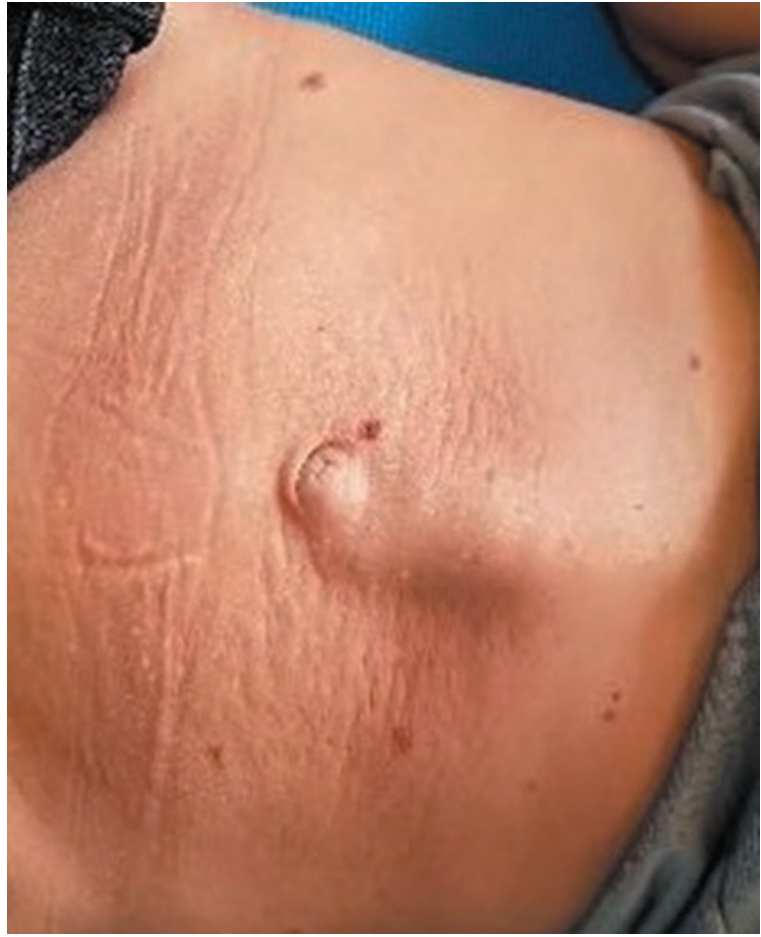

Figure 2. Bulging of the abdominal wall in DRA, a 26-year-old patient, 14 months postpartum (from the authors' own archives, reprinted with the patient's permission)

programme (strengthening of transversus abdominis muscles or rectus abdominis muscles), a postural training, education and training on appropriate mobility techniques and lifting techniques, methods strengthening transversus abdominis muscles (Pilates, functional training, the Tupler's technique exercises with or without abdominal splinting), the Noble technique (manual approximation of rectus abdominis muscles during a partial sit-up), a manual therapy (soft tissue mobilization, myofascial release), abdominal bracing and taping, the tubigrip or a corset. According to Keeller et al. [17] abdominal taping is often applied (in $40.8 \%$ respondents), but its effectiveness has not been confirmed so far.

Female patients are advised to avoid exercises which cause the bulging of the abdominal wall, exercises engaging oblique abdominal muscles, raising the lower limbs above the ground while lying on the back, abdominal sit-ups, crunches, intense coughing without abdominal support, as well as lifting heavy objects $[4,17]$.

In therapy, two optional treatment methods are used: engaging transversus abdominis muscles or rectus abdominis muscles. There is no agreement in existing data which of these methods is more effective in reducing IRD. According to one, exercises of transversus abdominis muscles (drawing-in exercise) are advised, whereas any exercises engaging the rectus abdominis muscles are forbidden as they may potentially increase IRD (abdominal sit-up/ curl-up, crunch exercise). The other method includes a crunch 
exercise in the supine position which activates the rectus abdominis muscles [4, 24]. According to Keller et al. [17] transversus abdominis training is usually applied (in $89.2 \%$ out of 296 respondents), although in $62.5 \%$ cases the Nobel technique engaging rectus abdominis muscles was applied. Few studies support these exercise suggestions. Mota et al. [29] compare the IRD measured in USG at rest and during drawing in and abdominal crunch exercises. An abdominal crunch exercise consistently produced a significant narrowing of the IRD, whereas a drawing-in exercise led to a small widening of the IRD. Similar results were reported by Sancho et al. [30]. According to Lee and Hodges [31] an abdominal curl-up results in the narrowing of the IRD in most women with DRA, although the preactivation of the transversus abdominis muscles reduces the narrowing of the IRD. However, the assessment of the linea alba tension expressed using the distortion index showed that the distortion index increased from rest during the curl-up, and the preactivation of the transversus abdominis muscle prevented the distortion of the LA. Proper tension of the linea alba is indispensable for the transfer of force between sides of the abdominal muscles, which results in a better control of abdominal content and better cosmetic outcome. It seems that the optimal strategy in the DRA therapy is combining the activity of the two kinds of abdominal muscles, but it still needs to be confirmed.

Apart from physiotherapy, there are trials of applying prolotherapy. Prolotherapy is the technique of injection of small amounts of an irritant solution into injured tissue (degenerated tendon insertions, joints, ligaments). Irritant solutions most often contain dextrose, phenol-glycerine-glucose (P2G), combinations of polidocanol, manganese, zinc, human growth hormone, pumice, ozone, glycerin, or phenol. The main aim of prolotherapy is to stimulate regenerative processes (growth of normal cells and tissues) [32]. Strauchman and Morningstar [33] report a case of decreasing the IRD from $2.7 \mathrm{~cm}$ to $0.5 \mathrm{~cm}$ after 7 prolotherapy sessions (injections were administered every two weeks). The solution used was a combination of dextrose, lidocaine, and methylcobalamin.

In the absence of efficacy of the conservative treatment, with high aesthetic and/or functional discomfort or the presence of hernia, surgical intervention is often used. The reduction of rectus abdominis muscles separation is also a part of abdominoplasty. Operative repair for DRA is controversial [34]. According to Emanuelsson et al. [12] the abdominal wall repair should be considered in patients with an IRD wider than $3 \mathrm{~cm}$. Brauman [35] states that this decision should be influenced primarily by the evaluation of the protrusion rather than diastasis. The operative procedures include the use of a retromuscular mesh or a double row of sutures with resorbable/non-resorbable suture material [12].

\section{SUMMARY}

Several authors have emphasized that in spite of the fact that DRA is both a common and significant clinical problem, little is known about its risk factors, prevention or management $[3,13,14]$. Undoubtedly, DRA may be treated as a cosmetic defect, and its presence may cause mental discomfort. Separation of the linea alba with the accompanying weakening of the anterior abdominal wall may lead to epigastric and umbilical hernias. In the light of studies results mentioned above, however, one cannot definitely confirm the link between DRA and such conditions as the pelvic floor dysfunction or lumbo-pelvic pain. Taking into account still unsatisfactory knowledge regarding risk factors, possible consequences and effective methods of preventive treatment there is still a need of conducting further research in this field.

\section{REFERENCES}

1. Coldron Y, Stokes MJ, Newham DiJ, et al. Postpartum characteristics of rectus abdominis on ultrasound imaging. Man Ther. 2008; 13(2): 112 -121, doi: 10.1016/j.math.2006.10.001, indexed in Pubmed: 17208034.

2. Axer $H$, Keyserlingk DG, Prescher A. Collagen fibers in linea alba and rectus sheaths. I. General scheme and morphological aspects. J Surg Res. 2001; 96(1): 127-134, doi: 10.1006/jsre.2000.6070, indexed in Pubmed: 11181006

3. Axer H, von Keyserlingk DG, Prescher A. Collagen fibers in linea alba and rectus sheaths. J Surg Res. 2001; 96(2): 239-245, doi: 10.1006/jsre.2000.6071, indexed in Pubmed: 11266279.

4. Benjamin DR, van de Water ATM, Peiris CL. Effects of exercise on diastasis of the rectus abdominis muscle in the antenatal and postnatal periods: a systematic review. Physiotherapy. 2014; 100(1): 1-8, doi: 10.1016/j. physio.2013.08.005, indexed in Pubmed: 24268942.

5. Liaw LJ, Hsu MJ, Liao CF, et al. The relationships between inter-recti distance measured by ultrasound imaging and abdominal muscle function in postpartum women: a 6-month follow-up study. J Orthop Sports Phys Ther. 2011; 41(6): 435-443, doi: 10.2519/jospt.2011.3507, indexed in Pubmed: 21289454.

6. Beer GM, Schuster A, Seifert B, et al. The normal width of the linea alba in nulliparous women. Clin Anat. 2009; 22(6): 706-711, doi: 10.1002/ca.20836, indexed in Pubmed: 19637295.

7. Chiarello C, Falzone L, McCaslin K, et al. The Effects of an Exercise Program on Diastasis Recti Abdominis in Pregnant Women. J Womens Health Phys Ther. 2005; 29(1): 11-16, doi: 10.1097/01274882200529010-00003.

8. Keshwani N, Hills N, McLean L. Inter-Rectus Distance Measurement Using Ultrasound Imaging: Does the Rater Matter? Physiother Can. 2016; 68(3): 223-229, doi: 10.3138/ptc.2015-36, indexed in Pubmed: 27909371.

9. Emanuelsson P, Dahlstrand U, Strömsten $U$, et al. Analysis of the abdominal musculo-aponeurotic anatomy in rectus diastasis: comparison of CT scanning and preoperative clinical assessment with direct measurement intraoperatively. Hernia. 2014; 18(4): 465-471, doi: 10.1007/s10029-0141221-0, indexed in Pubmed: 24488508.

10. van deWater ATM, Benjamin DR. Measurement methods to assess diastasis of the rectus abdominis muscle (DRAM): A systematic review of their measurement properties and meta-analytic reliability generalisation. Man Ther. 2016; 21: 41-53, doi: 10.1016/j.math.2015.09.013, indexed in Pubmed: 26474542.

11. Rett MT, Braga MD, Bernardes NO, et al. Prevalência de diástase dos músculos retoabdominais no puerpério imediato: comparação entre primíparas e multíparas. Rev Bras Fisioter. 2009; 13(4): 275-280, doi: 10.1590/s1413-35552009005000037.

12. Emanuelsson $P$, Gunnarsson $U$, Dahlstrand $U$, et al. Operative correction of abdominal rectus diastasis (ARD) reduces pain and improves abdominal wall muscle strength: A randomized, prospective trial comparing retromuscular mesh repair to double-row, self-retaining sutures. Surgery. 2016; 160(5): 1367-1375, doi: 10.1016/j.surg.2016.05.035, indexed in Pubmed: 27475817 
13. Fernandes da Mota PG, Pascoal AG, Carita Al, et al. Prevalence and risk factors of diastasis recti abdominis from late pregnancy to 6 months postpartum, and relationship with lumbo-pelvic pain. Man Ther. 2015; 20(1): 200-205, doi: 10.1016/j.math.2014.09.002, indexed in Pubmed: 25282439.

14. Sperstad JB, Tennfjord MK, Hilde G, et al. Diastasis recti abdominis during pregnancy and 12 months after childbirth: prevalence, risk factors and report of lumbopelvic pain. Br J Sports Med. 2016; 50(17): 1092-1096, doi: 10.1136/bjsports-2016-096065, indexed in Pubmed: 27324871.

15. Hilger T, Bagłaj M. Rzadko występujące przepukliny brzuszne u dzieci. Adv Clin Exp Med. 2006; 15(4): 733-740.

16. Cheesborough JE, Dumanian GA. Simultaneous prosthetic mesh abdominal wall reconstruction with abdominoplasty for ventral hernia and severe rectus diastasis repairs. Plast Reconstr Surg. 2015; 135(1): 268-276, doi: 10.1097/PRS.0000000000000840, indexed in Pubmed: 25539311.

17. Keeler J, Albrecht $M$, Eberhardt $L$, et al. Diastasis Recti Abdominis. J Womens Health Phys Ther. 2012; 36(3): 131-142, doi: 10.1097/jwh.0b013e318276f35f.

18. Roshan A, Khyati B, Ujwal Y, et al. Prevalence Of Diastasis Of Rectus Abdominis Muscle In Immediate Post-Partum Women Of Urban And Rural Areas. EJPRM. 2016; 3(5): 460-462.

19. Turan V, Colluoglu C, Turkyilmaz E, et al. Prevalence of diastasis recti abdominis in the population of young multiparous adults in Turkey. Ginekol Pol. 2011; 82(11): 817-821, indexed in Pubmed: 22384613.

20. Candido G, Lo T, Janssen PA. Risk factors for diastasis of the recti abdominis. J Assoc Chart Physiother Womens Health. 2005; 97: 49-54.

21. Parker M, Millar L, Dugan S. Diastasis Rectus Abdominis and Lumbo-Pelvic Pain and Dysfunction-Are They Related? J Womens Health Phys Ther. 2009; 33(2): 15-22, doi: 10.1097/01274882-200933020-00003.

22. Bø K, Hilde G, Tennfjord MK, et al. Pelvic floor muscle function, pelvic floor dysfunction and diastasis recti abdominis: Prospective cohort study. Neurourol Urodyn. 2017;36(3): 716-721, doi: 10.1002/nau.23005, indexed in Pubmed: 27037746.

23. Spitznagle TM, Leong FC, Van Dillen LR. Prevalence of diastasis recti abdominis in a urogynecological patient population. Int Urogynecol J Pelvic Floor Dysfunct. 2007; 18(3): 321-328, doi: 10.1007/s00192-0060143-5, indexed in Pubmed: 16868659.

24. Gitta S, Magyar Z, Tardi P, et al. How to Treat Diastasis Recti Abdominis with Physical Therapy: A Case Report. J Diseases. 2016; 3(2): 16-20, doi: 10.18488/journal.99/2016.3.2/99.2.16.20.
25. Acharry N, Kutty R. Abdominal exercise with bracing, a therapeutic efficacy in reducing diastasis-recti among postpartal females. Int J Physiother Res. 2015; 3(2): 999-1005, doi: 10.16965/ijpr.2015.122.

26. Walton L, Costa A, LaVanture D, et al. The effects of a 6 week dynamic core stability plank exercise program compared to a traditional supine core stability strengthening program on diastasis recti abdominis closure, pain, oswestry disability index (ODI) and pelvic floor disability index scores (PFDI). Phys Ther Rehabil. 2016; 3(1): 3, doi: 10.7243/2055-2386-3-3.

27. Awad M, Morsy M, Mohamed M, et al. Efficacy of Tupler Technique on Reducing Post Natal Diastasis Recti: A Controlled Study. Br J Appl Sci Technol. 2016; 12(1): 1-8, doi: 10.9734/bjast/2016/17876.

28. Khandale SR, Hande D. Effects of abdominal exercises on reduction of diastasis recti in postnatal women. Int J Health Sci Res. 2016; 6(6): 182-191.

29. Mota P, Pascoal AG, Carita Al, et al. The Immediate Effects on Inter-rectus Distance of Abdominal Crunch and Drawing-in Exercises During Pregnancy and the Postpartum Period. J Orthop Sports Phys Ther. 2015; 45(10): 781-788, doi: 10.2519/jospt.2015.5459, indexed in Pubmed: 26304639.

30. Sancho MF, Pascoal AG, Mota P, et al. Abdominal exercises affect inter-rectus distance in postpartum women: a two-dimensional ultrasound study. Physiotherapy. 2015; 101(3):286-291, doi: 10.1016/j.physio.2015.04.004, indexed in Pubmed: 26094117.

31. Lee D, Hodges PW. Behavior of the Linea Alba During a Curl-up Task in Diastasis Rectus Abdominis: An Observational Study. J Orthop Sports Phys Ther. 2016; 46(7): 580-589, doi: 10.2519/jospt.2016.6536, indexed in Pubmed: 27363572.

32. Hauser RA, Lackner JB, Steilen-Matias D, et al. A Systematic Review of Dextrose Prolotherapy for Chronic Musculoskeletal Pain. Clin Med Insights Arthritis Musculoskelet Disord. 2016; 9: 139-159, doi: 10.4137/CMAMD. S39160, indexed in Pubmed: 27429562

33. Strauchman M, Morningstar M. Prolotherapy Injections for Diastasis Recti: A Case Report. Case Reports Clinical Medicine. 2016; 05(09): 342-346, doi: 10.4236/crcm.2016.59052.

34. Siddiky AH, Kapadia CR. Laparoscopic plication of the linea alba as a repair for diastasis recti - a mesh free approach. J Surg Case Rep. 2010; 2010(5): 3, doi: 10.1093/jscr/2010.5.3, indexed in Pubmed: 24946321.

35. Brauman D. Diastasis recti: clinical anatomy. Plast Reconstr Surg. 2008 122(5): 1564-1569, doi: 10.1097/PRS.0b013e3181882493, indexed in Pubmed: 18971741. 